\title{
The neutral gas in the environs of the Geminga gamma-ray pulsar
}

\author{
E. Giacani ${ }^{a, *}$ E. M. Reynoso ${ }^{a, *}$ G. Dubner ${ }^{a, *}$ W. M. Goss ${ }^{b}$ \\ A. J. Green ${ }^{c}$ S. Johnston ${ }^{c}$ \\ ${ }^{a}$ Instituto de Astronomía y Física del Espacio (IAFE), CC 67, Suc. 28, 1428 \\ Buenos Aires, Argentina \\ ${ }^{\mathrm{b}}$ National Radio Astronomy Observatory, P.O. Box 0, Socorro, New Mexico \\ 87801, USA \\ ${ }^{\mathrm{c}}$ School of Physics, University of Sydney, NSW 2006, Australia
}

\begin{abstract}
We present a high-resolution $\left(24^{\prime \prime}\right)$ study of the HI interstellar gas distribution around the radio-quiet neutron star Geminga. Based on Very Large Array (VLA) and MPIfR Effelsberg telescope data, we analyzed a $40^{\prime} \times 40^{\prime}$ field around Geminga. These observations have revealed the presence of a neutral gas shell, $0.4 \mathrm{pc}$ in radius, with an associated $\mathrm{HI}$ mass of $0.8 \mathrm{M}_{\odot}$, which surrounds Geminga at a radial velocity compatible with the kinematical distance of the neutron star. In addition, morphological agreement is observed between the internal face of the HI shell and the brightest structure of Geminga's tail observed in X-rays. We explore the possibility that this morphological agreement is the result of a physical association.
\end{abstract}

Key words: neutron stars, neutron stars: Geminga, neutral hydrogen

\section{Introduction}

Neutron stars (NSs) have generally been detected as radio pulsars. In recent years, with the advent of high-energy instruments, several new classes of NSs have been discovered as unresolved X-ray sources. These include: anomalous X-ray pulsars, soft gamma ray repeaters, isolated neutron stars (also called "radio quiet neutron stars"), and compact central objects when the

\footnotetext{
* Member of the Carrera del Investigador Científico, CONICET

Email address: egiacani@iafe.uba.ar (E. Giacani).
} 
NSs are located inside supernova remnants (SNR). We have undertaken an investigation of the influence that these peculiar sources may have on the neutral gas in their environment. We have already studied three NSs: 1E 1207-5209 in G296.5+10.0 Giacani et al., 2000), RX J0822-4300 in Puppis A (Revnoso et al., 2003), and 1E 161345-5055 in RCW 103 (Revnoso et al., 2004). In all three cases there is a minimum in the HI emission, coincident with the location of the X-ray source, and at a radial velocity corresponding to the NS's kinematical distance. Hence, an association between the sources and their corresponding HI feature is quite probable. However, the physical mechanism that might give rise to such a phenomenon in the neutral HI gas remains unresolved.

We present here results based on HI observations obtained for the isolated Geminga pulsar (PSR J0633+1746). This source is known to pulsate from $\gamma$-rays to the optical band. In the radio domain, three groups independently reported the detection of weak pulsed radio emission at $102 \mathrm{MHz}$ (Malofeev and Malov, 1997; Kuzmin and Losovskii, 1997; Shitov and Pugachev, 1998). However, the detection of a radio continuum counterpart at other frequencies (74 and 330 $\mathrm{MHz}$ ) have so far been negative (Kassim and Lazio 1999 and references therein). Other groups have also failed to detect radio pulsations (Hankins, private communication). Geminga has a spin-down age of $3.4 \times 10^{5} \mathrm{yr}$ and a rotational energy loss of $E=3.2 \times 10^{34} \mathrm{erg} \mathrm{s}^{-1}$ (Bertsch et al., 1992). A distance of 160 pc and a transverse velocity of $130 \mathrm{~km} \mathrm{~s}^{-1}$ were determined from optical parallax measurements using the Hubble Space Telescope (Caraveo et al., 1996). Recently, Caraveo et al. (2003) reported the XMM-Newton X-ray detection of two elongated X-ray tails originating close to Geminga, which run parallel to the direction of motion of the pulsar and extend about $2^{\prime}$. The emission is produced by particles expelled by Geminga's strong magnetic field. The tails are the bright edges of the three-dimensional shockwave created by Geminga's motion. It has been suggested that Geminga is travelling almost directly transverse to the line of sight (Caraveo et al., 2003).

To constrain the physical properties of Geminga and its associated X-ray nebula, we have investigated their possible interactions with the surrounding interstellar HI gas.

\section{Observations}

A field of $40^{\prime} \times 40^{\prime}$ was observed in the HI $\left(\lambda 21 \mathrm{~cm}\right.$ line) with the $\mathrm{VLA}^{1}$ between 2001 September 3 and 2001 November 20 in the C, CnD and D

1 The VLA of the NRAO is a facility of the NSF, operated under cooperative agreement by Associated Universities, Inc. 
configurations for a total of more than 15 hours. The observations used the correlator in single-IF mode with on-line Hanning smoothing. Spectral information was detected in 256 channels, with a velocity resolution of $0.64 \mathrm{~km}$ $\mathrm{s}^{-1}$, over the velocity range from -82 to $+82 \mathrm{~km} \mathrm{~s}^{-1}$ (all velocities are relative to the LSR). Data reduction and calibration were carried out using the AIPS software package, following standard procedures. The $u-v$ visibilities from all the datasets were combined and the complete interferometric data cube has a $1 \sigma \mathrm{rms}$ noise of $\sim 2$ m. beam $^{-1}$.

To recover short spatial frequencies, the VLA data were combined in the $u-v$ plane with single dish observations from the $100 \mathrm{~m}$ MPIfR radio telescope at Effelsberg. A 201 channel correlator was used with a total effective bandwidth of $1.2 \mathrm{MHz}$, giving a velocity resolution of $1.3 \mathrm{~km} \mathrm{~s}^{-1}$ and an rms noise per channel of about $40 \mathrm{mK}$. These data are in units of brightness temperature, calibrated against the IAU standard position $\mathrm{S} 7\left(l=132^{\circ}, b=-1^{\circ}\right.$; Kalberla et al. (1980)). To combine the data sets, a factor of 1052 was used to convert the VLA flux densities from $\mathrm{Jy}_{\text {beam }}{ }^{-1}$ to brightness temperature in Kelvin. The angular resolution of the combined image is $24^{\prime \prime}$ and the $1 \sigma \mathrm{rms}$ noise in line-free channels is about $0.4 \mathrm{~K}$. The final cube covers the velocity range -68 to $+68 \mathrm{~km} \mathrm{~s}^{-1}$ with a velocity resolution of $1.3 \mathrm{~km} \mathrm{~s}^{-1}$.

\section{Results and Discussion}

We have examined the HI data cube looking for perturbations in the interstellar medium (ISM) that could be caused by the presence of the NS. HI emission features appear only in three velocity intervals. Most of the emission is near $15 \mathrm{~km} \mathrm{~s}^{-1}$, and is associated with the local spiral arm. The second emission peak is near $22 \mathrm{~km} \mathrm{~s}^{-1}$, which can be interpreted as emission from gas related to the Perseus spiral arm (Vogt, 1976; Caswell and Havnes, 1987). The only feature that could be related to Geminga appears in the third velocity range, between 1.3 and $4.5 \mathrm{~km} \mathrm{~s}^{-1}$.

At this third velocity range, Geminga is surrounded by an open HI shell (Figure 1). This incomplete ring, with an average angular radius of $9^{\prime} \quad(0.4 \mathrm{pc}$ at $160 \mathrm{pc})$, opens to the NW. The neutron star appears projected onto an HI void, which has a minimum at $\mathrm{RA}=06^{\mathrm{h}} 33^{\mathrm{m}} 53.0^{\mathrm{s}}, \mathrm{Dec}=17^{\circ} 47^{\prime} 47^{\prime \prime}(\mathrm{J} 2000)$, but is offset by about $2^{\prime}(0.09 \mathrm{pc})$ from the hole center. The close proximity of Geminga to both the Earth and the direction of the Galactic anticenter precludes a reliable estimate of the kinematical distance using the Galactic circular rotation model. However, the presence of this HI structure at such low velocities is consistent with the distance for Geminga, estimated by independent methods, and thus with a possible association. Moreover, the fact that Geminga is evolving inside a bubble is in good agreement with the very 


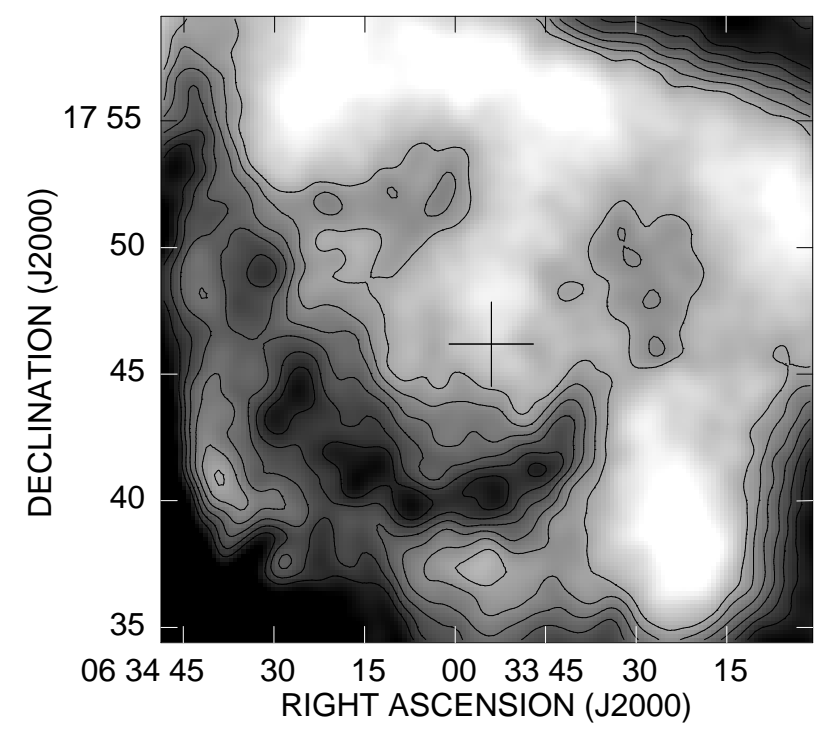

Fig. 1. Gray-scale and contour image of the HI emission towards Geminga averaged over the velocity interval $1.3-4.5 \mathrm{~km} \mathrm{~s}^{-1}$. The gray-scale ranges from 30 to $50 \mathrm{~K}$, while the contour levels are from 37 to $47 \mathrm{~K}$ in steps of $2 \mathrm{~K}$. The angular resolution has been smoothed to $50^{\prime \prime}$ to facilitate the identification of intense features. The cross indicates the position of Geminga.

low ambient density values $\left(\sim 0.06\right.$ to $0.15 \mathrm{~cm}^{-3}$ derived by Caraveo et al. 2003) to confine the X-ray bow-shock structure.

We have estimated the HI mass content of the shell by integrating the column density distribution within the velocity interval $(1.3,4.5) \mathrm{km} \mathrm{s}^{-1}$, assuming that the gas is optically thin. A total of $0.8 \mathrm{M}_{\odot}$ is obtained. It is difficult to explain the origin of the HI feature in connection with Geminga. Taking into account the proper motion of the pulsar and its age ( $\simeq \simeq 340000 \mathrm{yrs}$ ), Geminga was created in a supernova explosion 45 pc away from its current position. Therefore any possible association between the HI shell and Geminga's parent supernova can be ruled out.

In Figure 2a we display a comparison of the HI gas integrated between 1.3 and $4.5 \mathrm{~km} \mathrm{~s}^{-1}$ with the X-ray emission in the range 0.3 to $5 \mathrm{KeV}$ from Caraveo et al. (2003). There is excellent morphological agreement between the southern tail of the associated X-ray nebula and the internal border of the HI shell. This tail is the brighter of the two extensions in the X-ray source. It is possible that the higher HI density in this region has contributed to an enhancement of this X-ray feature.

The fact that the only HI minimum observed along the whole data cube agrees spatially and in velocity with Geminga's location, together with the excellent morphological agreement with the X-ray nebula, reinforces the possibility of 

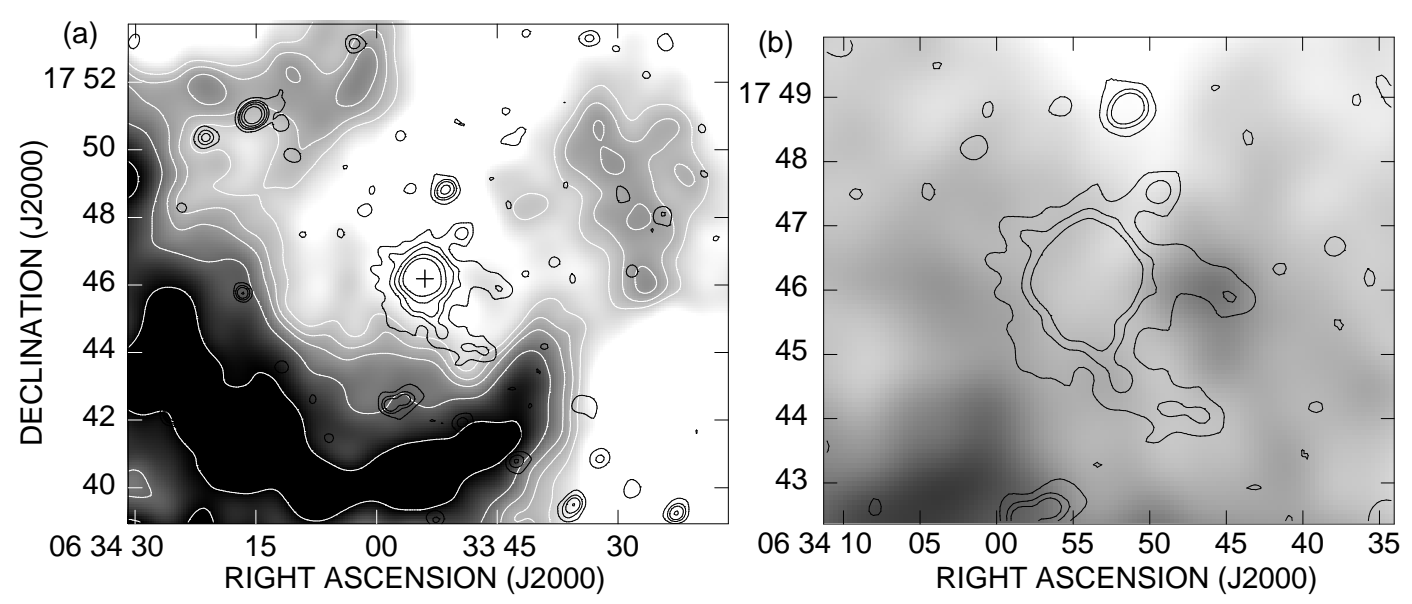

Fig. 2. (a) XMM-Newton X-ray data in the range 0.3 to $5 \mathrm{KeV}$ (black contours) from Caraveo et al. 2003, superimposed onto the integrated HI emission between 1.3 and $4.5 \mathrm{~km} \mathrm{~s}^{-1}$ (gray-scale and white contours). The cross indicates the position of Geminga. (b) The HI column density, integrated between 0 and $2 \mathrm{~km} \mathrm{~s}^{-1}$, is shown in gray-scale and ranges from $(1.0-1.6) \times 10^{20} \mathrm{~cm}^{-2}$. To show the location of the X-ray source, a few contours from the image of Caraveo et al. (2003) are included.

an association. We have conducted a literature search for early-type stars, open clusters, OB associations and HII regions that could have created HI shell-type features in the region, but none were found. Also, inspection of the IRAS (HIRES) images shows no infrared counterpart for this shell.

Figure $2 \mathrm{~b}$ displays the neutral hydrogen column density distribution, $\mathrm{N}_{\mathrm{H}}$, obtained from the integration of the foreground HI $\left(v \sim 0\right.$ to $\left.2 \mathrm{~km} \mathrm{~s}^{-1}\right)$. The absorbing column density slightly varies from $1.3 \times 10^{20} \mathrm{~cm}^{-2}$ in the direction of Geminga, to $1.5 \times 10^{20} \mathrm{~cm}^{-2}$ towards the northern X-ray tail. It is significant that the HI column density does not increase between the tails, indicating that the observed morphology of the X-ray source is real and not a consequence of variable X-ray absorption due to the intervening HI column.

Based on the line-free channels of our $1.4 \mathrm{GHz}$ cube, we have produced an image of the radio continuum at this frequency in the direction of Geminga with $24^{\prime \prime}$ angular resolution. We have not detected any radio continuum source, either point-like or extended, down to a noise level of $0.14 \mathrm{mJy}_{\text {beam }}{ }^{-1}$. We can speculate that if a radio pulsar wind nebula (PWN) existed, at least the same size as the X-ray nebula, then the maximum flux density at $1.4 \mathrm{GHz}$, consistent with our non-detection (at the $3 \sigma$ noise level) would be $54 \mathrm{mJy}$. For a typical PWN spectral index of $\alpha=-0.3\left(\mathrm{~S} \propto \nu^{\alpha}\right)$, the broadband radio luminosity $\mathrm{L}_{\mathrm{r}}$ between $10^{7}$ and $10^{11} \mathrm{~Hz}$ would be $6 \times 10^{28} \mathrm{erg} \mathrm{s}^{-1}$, corresponding to an efficiency $\epsilon \equiv \mathrm{L}_{\mathrm{r}} / \dot{E} \sim 10^{-6}$. This value is significantly lower than those found for other radio PWN but comparable to that of PSR B1706-44 (Giacani et al., 2001), which is a young isolated pulsar with a pulsed gamma-ray source. Since 
Geminga is an old pulsar, the production efficiency of synchrotron radiation would be reduced. We have also processed archival VLA data from B-array observations. The image, with a resolution of $4^{\prime \prime}$ and an rms noise of $31 \mu \mathrm{Jy}$ beam $^{-1}$, shows no counterpart.

Summing up , the present work is the first high resolution, high sensitivity survey of the neutral interstellar gas around Geminga. These observations have revealed that the pulsar lies inside an interstellar bubble, probably preexistent. The good morphological agreement between the brightest structure of Geminga's X-ray tail and the wall of the bubble suggests the existence of interaction between them. A theoretical investigation is necessary to understand the mechanism through which the HI/X-ray interaction have enhanced the X-ray tail.

The authors would like to thank Peter Kalberla for carrying out the observations with the $100 \mathrm{~m}$ telescope of MPIfR (Max-Planck- Institut für Radioastronomie) at Effelsberg and for processing those data. This research was funded through CONICET (Argentina) grant PIP2136/00 and grant UBACYT (Argentina) A055.

\section{References}

Bertsch, D. L., Brazier, K. T., Fichtel, C. E., et al. Pulsed high-energy gammaradiation from Geminga (1E0630 + 178). Nature 357, 306-307, 1992.

Caraveo, P. A., Bignami, G. F., Mignani, R., Taff, L. G. Parallax observations with the Hubble Space Telescope yield the distance to Geminga. ApJ 461, L91-L94, 1996.

Caraveo, P. A., Bignami, G. F., DeLuca, A., et al. Geminga's tails: a pulsar bow shock probing the interstellar medium. Science 301, 1345-1348, 2003.

Caswell, J. L., Haynes, R. F. Southern H II regions - an extensive study of radio recombination line emission 1987. A\&A 171, 261-276, 1987.

Giacani, E. B., Dubner, G. M., Green, A. J., et al. The interstellar matter in the direction of the supernova remnant G296.5+10.0 and the central X-Ray source 1E 1207.4-5209. AJ 119, 281-291, 2000.

Giacani, E. B., Frail, D. A., Goss, W. M., Vieytes, M. Pulsar wind nebulae around the southern pulsars PSR B1643-43 and PSR B1706-44. AJ 121, 3133-3137, 2001.

Kalberla, P. M. W., Mebold, U., Reich, W. Time variable $21 \mathrm{~cm}$ lines and the stray radiation problem. A\&A 82, 275-286, 1980.

Kassim, N. E., Lazio, T. J. W. Upper limits on the continuum emission from Geminga at 74 and 326 MHZ 1999. ApJ 527, L101-L104, 1999.

Kuzmin, A. D., Losovskii, B. Y. Detection of the radio pulsar PSR J0633 + 1746 in Geminga. Astronomy Letters 23, 283-285, 1997. 
Malofeev, V. M., Malov, O. I. Detection of Geminga as a radio pulsar. Nature 389, 697-699, 1997.

Reynoso, E. M., Green, A. J., Johnston, S., et al. Observations of the neutral hydrogen surrounding the radio-quiet neutron star RX J0822-4300 in Puppis A. MNRAS 345, 671-677, 2003.

Reynoso, E. M., Green, A. J., Johnston, S., et al. Influence of the neutron star 1E 161348-5055 in RCW 103 on the surrounding medium. PASA 21, 82-88, 2004.

Shitov, Y. P., Pugachev, V. D. The radio pulsar Geminga. New Astronomy 3, 101-109, 1998.

Vogt, N. Galactic structure in monoceros : a photometric study of luminous stars. A\&A 53, 9-14, 1976. 